\title{
Flucht in den Untergrund: zur Situation der jüdischen Bevölkerung in Deutschland
}

\author{
1941-1945
}

Claudia Schoppmann

Am 23. Oktober 1941 verfügten die Nationalsozialisten ein Auswanderungsverbot für die jüdische Bevölkerung im Deutschen Reich. Der Zeitpunkt war bewusst gewählt: Wenige Tage zuvor, am 18. Oktober, hatten sie mit der reichsweiten Deportation der jüdischen Minderheit begonnen, die zunächst nach Polen, Weißrussland und ins Baltikum ging, ab 1942 vor allem nach Auschwitz und Theresienstadt. Damit war Deutschland für die jüdische Bevölkerung, die ihre Heimat nicht rechtzeitig hatte verlassen können, zur tödlichen Falle geworden. Betroffen waren alle Menschen, die - unabhängig von ihrem Selbstverständnis oder Glaubensbekenntnis aufgrund der »Nürnberger Gesetze« zu Juden erklärt und im Lauf der Jahre mit Hilfe von über 1.900 Verordnungen Schritt für Schritt ausgegrenzt, entrechtet und verfolgt worden waren. ${ }^{1}$ Der Höhepunkt ihrer Stigmatisierung war mit der Polizeiverordnung vom 19. September 1941 erreicht, die die öffentliche Kennzeichnung aller Juden mit einem gelben Stern anwies. Danach war es nicht mehr möglich, antijüdische Maßnahmen zu umgehen, indem man etwa in einen anderen Stadtteil ging, in welchem einen niemand kannte.

Bis zum Ende der NS-Herrschaft gab es nur zwei Möglichkeiten, sich der Deportation zu entziehen: durch die Flucht in eine illegale Existenz oder ins Ausland. Letzteres glückte nur in wenigen Fällen. Fast alle Länder, die Deutschland umgaben, waren entweder besetzt oder verbündet und kamen daher als Fluchtziel nicht in Frage. Die Schweiz, als einziges angrenzendes neutrales Land, hatte aufgrund ihrer judenfeindlichen Flüchtlingspolitik schon seit August 1938 Personen ohne Visum zurückgewiesen. Vier Jahre später, ab August 1942, verwehrte man den Verfolgten - schätzungsweise mehreren Tausend Juden - generell die Einreise. Um auf

1 I Joseph Walk (Hg.): Das Sonderrecht für Juden im NS-Staat, Heidelberg: C. F. Müller 1981. 
illegalem Weg in die Schweiz zu gelangen, benötigte man Geld, gefälschte Papiere, die einer Kontrolle standhalten konnten, und vor allem Kontakte zu Ortskundigen in den Grenzregionen, die bereit und in der Lage waren, einen über die Grenze zu schleusen. ${ }^{2}$ Doch auch nach geglücktem Grenzübertritt wurden etliche Flüchtlinge wieder des Landes verwiesen - meist mit tödlichen Folgen. ${ }^{3}$

Die 164.000 Juden, die vor Beginn der Deportationen im Herbst 1941 noch in Deutschland lebten, waren durch Emigration und nationalsozialistische Repressionen eine isolierte, verarmte und überalterte Gruppe. Dabei überwog die Anzahl der Frauen die der Männer um 32.000, ${ }^{4}$ was neben Restriktionen seitens der Exilländer (die qualifizierte männliche Arbeitskräfte bevorzugten) auch darauf zurückzuführen ist, dass Frauen häufig ihre Eltern nicht allein zurücklassen wollten. 1941 lebten 44 Prozent aller im Reich verbliebenen Juden - 73.000 Menschen - in Berlin, wo sie in Rüstungsbetrieben Zwangsarbeit leisten mussten. Nach 1933 waren viele Juden aus anderen Städten und vom Land in die Reichshauptstadt gezogen, weil sie hofften, von hier aus ihre Auswanderung besser betreiben zu können, von Institutionen der jüdischen Selbsthilfe unterstützt zu werden und in der Anonymität der Großstadt vor Repressionen geschützter zu sein.

$\mathrm{Zu}$ Beginn der Deportationen waren die tödlichen Folgen nicht bekannt - und auch kaum vorstellbar. Verschleiernd war offiziell stets von »Umsiedlung«, »Abwanderung« oder »Evakuierung« die Rede. Erst im Laufe des Jahres 1942 verbreiteten sich in Deutschland Gerüchte über die Massenerschießungen, die Konzentrations- und Vernichtungslager. ${ }^{5}$ Die Vorstellung einer fabrikmäßig betriebenen Ermordung erschien jedoch so ungeheuerlich, dass selbst diejenigen, die etwa von einem Soldaten auf Heimaturlaub oder durch das verbotene Abhören eines ausländischen Senders von den wahren Vorgängen erfahren hatten, sich sträubten, dies zu glauben. Angesichts der enormen Probleme, die ein Leben im Untergrund bedeu-

2 | Siehe Claudia Schoppmann: »Fluchtziel Schweiz. Das Hilfsnetz um Luise Meier und Josef Höfler«, in: Wolfgang Benz (Hg.), Überleben im Dritten Reich. Juden im Untergrund und ihre Helfer, München: C. H. Beck 2003, S. 203-217; Franco Battel: »Wo es hell ist, dort ist die Schweiz«. Flüchtlinge und Fluchthilfe an der Schaffhauser Grenze zur Zeit des Nationalsozialismus, Zürich: Chronos 2000.

3 | Dies zeigt etwa das Beispiel von Kurt und Irmgard Wohlmuth aus Berlin, die mit Hilfe von Marta Mierendorff in Vorarlberg (Österreich) über die Schweizer Grenze gelangten. Sie wurden jedoch von Grenzbeamten an die Deutschen ausgeliefert und später in Berlin erschossen. Vgl. Marta Mierendorff: »Von der Schweiz ausgeliefert, in Deutschland erschossen. Fam. Wohlmuth und Salomon, Deutschmeisterstr. 1«, in: Thea Koberstein/Norbert Stein (Hg.), Juden in Lichtenberg mit den früheren Ortsteilen in Friedrichshain, Hellersdorf und Marzahn, Berlin: Edition Hentrich 1995, S. 166-168.

4 । Monika Richarz (Hg.): Jüdisches Leben in Deutschland, Bd. 3, Stuttgart, München: DVA 1982, S. 61.

5 | Zur Kenntnis der Deutschen über die Vernichtungspolitik der Nationalsozialisten siehe Peter Longerich: »Davon haben wir nichts gewusst!« Die Deutschen und die Judenverfolgung 1933-1945, München: Siedler 2006; David Bankier: »Was wussten die Deutschen vom Holocaust?«, in: Kosmala/Schoppmann (Hg.), Überleben im Untergrund (2002), S. 63-87. 
tete, tauchten viele Jüdinnen und Juden erst unter, als Angehörige bereits deportiert worden waren oder ihre eigene Verschleppung unmittelbar bevorstand. Auch psychologisch war der Schritt in die Illegalität schwierig, bedeutete dies doch, die letzten Reste einer geregelten Existenz - wie eingeschränkt auch immer sie bereits war - zu verlieren. Da sie zu Beginn der Deportationen nicht ahnen konnten, wie lange der Krieg noch dauern würde, war dies ein Entschluss auf unbestimmte Dauer. Erst Anfang 1943, als sich die Niederlage der Wehrmacht bei Stalingrad abzeichnete, war ein Sieg der Alliierten und damit ein Ende der NS-Herrschaft überhaupt vorstellbar.

Wurde die Verschleppung in den ersten Monaten seit Beginn der reichsweiten Deportationen im Oktober 1941 noch schriftlich mitgeteilt, so erfolgten die Verhaftungen ab Ende 1942 unangekündigt. Letztes Signal zum Untertauchen war in vielen Fällen die Großrazzia Ende Februar 1943, die als »Fabrik-Aktion« bekannt wurde. Damals sollten alle noch im Reichsgebiet lebenden Juden deportiert werden, ausgenommen diejenigen, die durch ihre Ehe mit einem nichtjüdischen Partner vorläufig noch geschützt waren. So wurden am 27. Februar 1943 Tausende von Zwangsarbeiterinnen und Zwangsarbeitern in Rüstungsbetrieben, die bis dahin wegen ihrer Kriegswichtigkeit ihren Beschäftigten noch einen gewissen Schutz geboten hatten, in den Fabriken, zuhause oder auf der Straße verhaftet und wenige Tage später deportiert. Allein in Berlin sollen bei dieser Razzia mehr als 4.000 Verfolgte untergetaucht sein. ${ }^{6}$ Insgesamt wurden im März 1943 etwa 11.000 Jüdinnen und Juden verschleppt, von denen ungefähr zwei Drittel aus Berlin waren. Was es konkret bedeutete, monate- und jahrelang im Verborgenen bzw. mit einer vermeintlich »arischen« Identität zu leben, soll an zwei Beispielen verdeutlicht werden.

\section{Als $>U$-Boot in der Reichshauptstadt}

Gertrude Sandmann (1893-1981) ${ }^{7}$ steht stellvertretend für die vielen, die in den Jahren 1941 bis 1945 in der Reichshauptstadt untergetaucht sind - schätzungsweise waren es 5.000 bis 7.000 Personen. ${ }^{8}$ Die politisch links eingestellte Künstlerin, die einst Privatunterricht bei Käthe Kollwitz genommen hatte und dem Verein Berliner Künstlerinnen angehörte, erkannte früh, dass die Machtübernahme der Nationalso-

6 | Das heißt, dass damals etwa jeder Dritte der in Berlin für die Deportation Bestimmten (= 11.000 Personen) sich dem Zugriff der Verfolger entzog. Siehe Wolf Gruner: Widerstand in der Rosenstraße: die Fabrik-Aktion und die Verfolgung der »Mischehen« 1943, Frankfurt/ Main: S. Fischer 2005; Claudia Schoppmann: »Die >Fabrikaktion〈 in Berlin: Hilfe für untergetauchte Juden als Form humanitären Widerstands«, in: Zeitschrift für Geschichtswissenschaft 2 (2005), S. 138-148.

7 | Claudia Schoppmann: Zeit der Maskierung. Lebensgeschichten lesbischer Frauen im $»$ Dritten Reich«, Frankfurt/Main: S. Fischer 1998, S. 79-96.

8 | Gerald Reitlinger (in: Die Endlösung. Hitlers Versuch der Ausrottung der Juden Europas 1939-1945, Berlin: Colloquium 1956, S. 180) nannte für Mitte 1943 gar 9.000 Untergetauchte für Berlin. Siegmund Weltlinger ging 1954 von »etwa 5.000« aus (zitiert nach Kurt R. Grossmann: Die unbesungenen Helden. Menschen aus Deutschlands dunklen Tagen, Berlin: 
zialisten nichts Gutes verheißen konnte, und ging in die Schweiz. 1934 musste sie jedoch nach Deutschland zurückkehren, da ihr die Schweiz keine Aufenthalts- und Arbeitserlaubnis mehr gewährte. Im selben Jahr wurde sie wegen »nichtarischer Abstammung« aus dem Reichsverband bildender Künstler ausgeschlossen und erhielt Berufsverbot. Noch einmal entschloss sie sich zur Flucht. Es gelang ihr, eines der raren Visa für Großbritannien zu ergattern. Doch sie brachte es nicht übers Herz, ihre inzwischen schwerkranke, verwitwete Mutter in Berlin allein zurückzulassen. Als diese im Oktober 1939 starb, war das Visum wegen des Kriegsbeginns wertlos geworden.

Im November 1942 war Gertrude Sandmann, da sie aus gesundheitlichen Gründen keine Zwangsarbeit leisten konnte und sich somit nicht als >nützlichく erwies, unmittelbar von der Deportation bedroht. Sie ging das Wagnis ein und tauchte unter. Am 21. November 1942 floh sie aus ihrer eigenen Wohnung und hinterließ der Gestapo, die bald alles ausrauben sollte, einen Abschiedsbrief, in dem sie ihren Selbstmord ankündigte. ${ }^{9}$ Um diesen Entschluss glaubwürdig aussehen zu lassen, musste sie alles in ihrer Wohnung zurücklassen, inklusive der Lebensmittelkarten, die Juden ohnehin nur ein Fünftel der üblichen Ration zugestanden. Ohne die Hilfe ihrer Lebensgefährtin und weiterer Freundinnen wäre dieser Schritt unmöglich gewesen. Die Kunstgewerblerin Hedwig Koslowski, mit der Gertrude Sandmann seit 1927 liiert war, ließ sie nicht im Stich - keine Selbstverständlichkeit in einer Zeit, in der viele »Mischehen« unter dem äußeren Druck zerbrachen. Der relative Schutz, den eine »Mischehe« dem jüdischen Partner vorläufig vor der Deportation gewährte, galt selbstverständlich nicht für homosexuelle Partnerschaften, verstießen diese doch gegen das »gesunde Volksempfinden«. Homosexuelle Beziehungen zwischen Männern waren darüber hinaus häufig strafrechtlicher Verfolgung ausgesetzt. $^{10}$

Hedwig Koslowski organisierte einen Unterschlupf bei der befreundeten Familie Grossmann - der Mann war als Soldat eingezogen - in Berlin-Treptow. In einer winzigen Kammer hielt Gertrude Sandmann sich verborgen, lebte von dem, was Frau Grossmann von ihrer Essensration abzweigte und was Hedwig Koslowski besorgen konnte. Was es heißt, monate-, ja jahrelang im Versteck zu leben, hat Anne Frank in ihrem Tagebuch eindringlich beschrieben. Auch Gertrude Sandmann

Arani Verlags GmbH 1957, S.104-106, hier S. 104). Bei den Recherchen zum Berliner Gedenkbuch ließen sich in 3.500 Fällen in den Quellen Hinweise auf temporäre Illegalität finden (FU Berlin [Hg.]: Gedenkbuch Berlins der jüdischen Opfer des Nationalsozialismus, Berlin: Edition Hentrich 1995, S. 1407).

9 | Als die Deportationsbefehle eintrafen, entschieden sich 3.000 bis 4.000 überwiegend alte Menschen dafür, in dieser für sie ausweglos scheinenden Situation zuhause und von eigener Hand, oft gemeinsam mit Angehörigen, zu sterben. Suizid wurde zum Massenphänomen, weshalb der Schwarzmarktpreis für Veronal stark anstieg. Siehe Anna Fischer (Hg.): Erzwungener Freitod. Spuren und Zeugnisse in den Freitod getriebener Juden der Jahre 1938-1945 in Berlin, Berlin: Textpunkt Verlag 2007.

10 | Siehe etwa Andreas Pretzel/Gabriele Rossbach (Hg.): „Wegen der zu erwartenden hohen Strafe ...«. Homosexuellenverfolgung in Berlin 1933-1945, Berlin: Rosa Winkel 2000. 
musste bei Abwesenheit ihrer Helferin jedes Geräusch in der hellhörigen Wohnung vermeiden, durfte nicht ans Fenster treten und die Wohnung nie verlassen, auch nicht während der schwersten Bombenangriffe, denen sie schutzlos ausgeliefert war. Nach der Entwarnung, wenn der Luftschutzwart seinen Kontrollgang durch die Wohnungen machte, blieb der zierlichen Frau nichts anderes übrig, als sich schnell in einem Schreibtisch zu verstecken und zu hoffen, dass kein plötzlicher Hustenanfall sie verriet.

Im Sommer 1944, nach anderthalb Jahren des Eingesperrtseins, wurde die beengte Situation immer unerträglicher. Auch mochte Gertrude Sandmann ihre Helferin nicht länger gefährden, denn wenn entdeckt worden wäre, dass sie eine Jüdin versteckte, hätte ihr KZ-Haft gedroht. Und was wäre dann aus ihrer minderjährigen Tochter geworden? Wieder gelang es Hedwig Koslowski, einen Unterschlupf zu besorgen, diesmal in einer unbewohnten Laube in Biesdorf, am Stadtrand von Berlin. Feuer und Licht durfte sie wegen der Nachbarn nicht machen. Hedwig Koslowski und eine langjährige Freundin Gertrude Sandmanns aus den 1920er Jahren, Susy Hermans, versorgten die Untergetauchte mit Essen. Da sie längst nicht mehr zeichnen konnte, deklamierte sie Gedichte und trainierte ihr Gedächtnis, um nicht den Verstand zu verlieren. Im Herbst 1944 machte die einsetzende Kälte eine erneute Flucht nötig: Diesmal nahm Hedwig Koslowski die Freundin mit in ihre eigene Wohnung im Berliner Bezirk Schöneberg, die sie mit einer anderen Kunstgewerblerin teilte. Dort erlebte Gertrude Sandmann, auf 70 Pfund abgemagert, die Befreiung. Bis zu ihrem Lebensende konnte ein unerwartetes Klopfen an der Tür oder das Klingeln des Telefons sie in Angst und Schrecken versetzen und nächtliche Alpträume heraufbeschwören.

Die Versuche, »illegal« zu überleben, beschränkten sich nicht auf Berlin. ${ }^{11} \mathrm{Je}-$ doch waren in anderen Städten, etwa in Frankfurt/Main, der Stadt mit der zweitgrößten jüdischen Gemeinde, ${ }^{12}$ die Deportationen meist schon im Herbst 1942 abgeschlossen - also in etwa zu dem Zeitpunkt, als sich die Gerüchte über das Schicksal der Deportierten zu verbreiten begannen. Das ist einer der Gründe, weshalb außerhalb Berlins nur verhältnismäßig wenigen Verfolgten die Flucht in den Untergrund gelang. Als Ende 1944/Anfang 1945 auch die bislang meist noch geschützten Juden in »Mischehe« und viele »Mischlinge« verschleppt werden sollten, kam es zu weiteren Fluchtversuchen in Berlin und anderswo.

11 | $\mathrm{Zu}$ Berlin sind besonders viele Quellen überliefert, etwa die Anträge Überlebender auf Anerkennung als »Opfer des Faschismus « sowie die Unterlagen der Ehrungsinitiative »Unbesungene Helden« des Westberliner Senats. Siehe Dennis Riffel: Unbesungene Helden. Die Ehrungsinitiative des Berliner Senats 1958 bis 1966, Berlin: Metropol 2007.

12 I Siehe Monica Kingreen: "Verfolgung und Rettung in Frankfurt am Main und der Rhein-Main-Region«, in: Kosmala/Schoppmann (Hg.), Überleben im Untergrund (2002), S. $167-190$. 


\section{Versteckt bei Bauern im Münsterland}

Das zweite Beispiel gehört zu den wenigen dokumentierten Fällen aus ländlicher Region, ${ }^{13}$ und es zeigt die besondere Problematik, in der sich jüdische Paare und Familien befanden - machte doch ein Untertauchen in den meisten Fällen eine Trennung auf unbestimmte Zeit notwendig.

Marga und Siegmund Spiegel und ihre 1938 geborene Tochter Karin lebten im westfälischen Ahlen nahe Hamm. ${ }^{14} 1939$ mussten sie in ein »Judenhaus« nach Dortmund ziehen, wo Siegmund Spiegel Zwangsarbeit in einer Zeche leistete. Sein Chef schützte ihn und die anderen jüdischen Arbeiter davor, frühzeitig deportiert $\mathrm{zu}$ werden, indem er sie immer wieder mit der Begründung reklamierte, dass er keine Nichtjuden für die schwere Arbeit bekommen könne. Als Spiegels Ende Februar 1943 die Aufforderung der Gestapo bekamen, sich am folgenden Morgen angeblich zur Überprüfung der Arbeitspapiere - am Sammelplatz einzufinden, ahnte Siegmund Spiegel, was ihnen in Wirklichkeit drohte. In den Monaten zuvor war er immer wieder verbotenerweise mit dem Fahrrad ins Münsterland gefahren, zu Bauern, die den einstigen Viehhändler von früher kannten und schätzten. Dort hatte er Lebensmittel »organisiert«, aber auch nach Versteckmöglichkeiten gesucht. Einer der Bauern, Hubert Pentrop aus dem Dorf Nordhausen, hatte von einem Nachbarssohn von Erschießungen von Juden in Polen gehört und sagte daraufhin zu Siegmund Spiegel: »Wenn sie dich nach Polen schicken wollen, geh nicht mit. Von dort hört man nichts Gutes. Komm zu mir, ich verstecke dich! « ${ }^{15}$ Ende Februar 1943 war dieser Moment gekommen. Das bedeutete jedoch, dass die Familie sich nun trennen musste: Siegmund Spiegel wurde bei Familie Pentrop versteckt, in einem kleinen, unbeheizten Verschlag, den er nur nachts gelegentlich verlassen konnte. Für ihn als Mann im »wehrfähigen« Alter wäre es zu gefährlich gewesen, sich offen zu zeigen, zumal er keine falschen Papiere besaß. Auch war Siegmund Spiegel aufgrund seiner früheren Tätigkeit als Viehhändler in der Umgebung bekannt. Für seine Frau und Tochter bat er die Familie Aschoff im nahe gelegenen Dorf Herbern - sie waren ebenfalls frühere Geschäftskunden - um Hilfe. Maria Aschoff hatte von Soldaten Gerüchte über die Konzentrationslager im Osten gehört und ahnte, welche Gefahr den Deportierten dort drohte. Sie und ihr Mann Heinrich waren bereit, die ihnen bis dahin unbekannte Frau Spiegel und ihre Tochter bei sich aufzunehmen. Von ihren acht Kindern weihten Aschoffs nur die ältesten beiden Töchter ein.

Ein regelrechtes Verstecken war mit der fünfjährigen Karin auf Dauer nicht möglich. Frau Spiegel wurde deshalb auf dem Hof als Frau »Krone« aus Dort-

13 | Vgl. Claudia Schoppmann: »)Da packte mich das Mitleid und ich beherbergte ihn<. Tödliche Folgen für einen Bauern im Odenwald«, in: Beate Kosmala/Claudia Schoppmann (Hg.), Sie blieben unsichtbar. Zeugnisse aus den Jahren 1941 bis 1945, Berlin: Gedenkstätte Deutscher Widerstand 2006, S. 50-57.

14 | Zum Folgenden siehe Marga Spiegel: »Retter in der Nacht«. Wie eine jüdische Familie im Münsterland überlebte, Köln: Pahl Rugenstein 1987.

15 | Ebd., S. 92. 
mund ausgegeben, die wegen der Bombenangriffe auf die Großstädte »evakuiert« sei. Dass sie blond und blauäugig war, erleichterte die Tarnung. Ihrer Tochter hatten Spiegels zuvor beigebracht, dass sie in Zukunft »Krone« heiße und ihr Vater als Soldat an der Front sei. Die Situation war keineswegs ungefährlich, da immer wieder auch Fremde auf den Hof kamen. Einmal wurde Marga Spiegel von einer Besucherin aus Dortmund erkannt. Jedoch schwieg diese Frau und behielt ihre Entdeckung für sich.

Auch Siegmund Spiegel war in seinem Versteck bei Pentrops nicht sicher. Dort wurde er im November 1943 von einem Hitlerjungen entdeckt und musste schnellstens verschwinden. Aufnahme fand er erneut bei Bauern in der Nähe. Trotz der gerade erst überstandenen Gefahr nahmen Pentrops später auch Frau Spiegel und ihre Tochter bei sich auf. Eines Nachts standen plötzlich zwei Polizisten vor der Tür, die nach einer nicht gemeldeten Person suchten. Bauer Pentrop blieb gefasst und versicherte geistesgegenwärtig, dass er keine unangemeldete Person beherberge. Die Polizisten gaben sich glücklicherweise mit dieser Auskunft zufrieden und sahen davon ab, das Haus zu durchsuchen. Aus Angst vor Entdeckung mussten Marga und Karin Spiegel ihr Versteck noch mehrmals wechseln - ebenso wie Siegmund Spiegel. Stets konnten sie sich auf die Bauernfamilien - insgesamt waren es fünf - verlassen, die ihren katholischen Glauben ernst nahmen und bereit waren, dem Gebot der Nächstenliebe auch unter solch schwierigen Bedingungen zu folgen.

Schließlich konnte sich Marga Spiegel im Herbst 1944 - wie manch anderer Illegaler auch - falsche Papiere besorgen, indem sie nach einem schweren Bombenangriff auf Münster auf dem dortigen Polizeirevier behauptete, ihre Dokumente seien bei einem Luftangriff vernichtet worden. Es gelang ihr tatsächlich, einen Ausweis als »Bombenopfer« zu erhalten und die kommenden Monate bis zur Befreiung durch die Amerikaner zu überstehen. Spiegels blieben nach dem Krieg in Deutschland, obwohl zahlreiche Familienangehörige ermordet worden waren. Es war wohl die von Nichtjuden erfahrene lebensrettende Hilfe, die es ihnen ermöglichte, im Land der Täter zu bleiben.

\section{Flucht vor der Deportation}

Die beiden Beispiele zeigen stellvertretend, mit welchem Mut jüdische Menschen ihr Leben zu retten versuchten; sie widerlegen das Bild vom wehrlosen Opfer, das sich lange Zeit nach Kriegsende gehalten hat. Mit der Flucht vor der Deportation widersetzten sie sich den Befehlen eines totalitären Regimes, dessen Absicht es war, die jüdische Bevölkerung Europas zu vernichten.

Trotz des enormen Verfolgungsdrucks und der besonderen Gefährdung gab es auch zahlreiche Versuche gegenseitiger Unterstützung und politischen Widerstandes von Jüdinnen und Juden. Erwähnt sei hier eine Gruppe jüdischer Jugendlicher, die sich »Chug Chaluzi« (hebräisch für »Pionierkreis«) nannte. Sie waren nach der »Fabrik-Aktion« Ende Februar 1943 in Berlin untergetaucht und halfen sich gegenseitig. Die Gruppe verstand die Rettung jüdischen Lebens als eine Form des politischen Widerstandes: »Mit jedem geretteten Leben bekämpfen wir 
Hitler!«war ihre Parole. ${ }^{16}$ Eine entscheidende Rolle bei der Organisation des Widerstandes spielte die 40-jährige Edith Wolff, die nach den Nürnberger Rassegesetzen als »Mischling 1. Grades« galt und deshalb zunächst nicht von der Deportation bedroht war. Sie nutzte ihre größere Bewegungsfreiheit, um den bis zu 40 Jugendlichen beim Sprung »in den luftleeren Raum«, wie sie es nannte, zu helfen. Im Juni 1943 wurde Edith Wolff von der Gestapo gefasst. Sie überlebte 17 Gefängnisse und das KZ Ravensbrück. Da sie trotz Folter niemanden verraten hatte, konnten die meisten Jugendlichen überleben.

Als größte jüdische Widerstandsgruppe gilt der kommunistisch und sozialistisch orientierte Freundeskreis um Herbert und Marianne Baum in Berlin, der antifaschistische Flugblätter verteilte und Illegalen half. Nach einem Brandanschlag im Mai 1942 auf die Propagandaausstellung »Das Sowjetparadies« wurden zahlreiche Mitglieder der Gruppe, der zeitweilig bis zu hundert Jugendliche angehörten, verhaftet und hingerichtet. ${ }^{17}$

Eine höchst ungewöhnliche Allianz von jüdischen und nichtjüdischen Regimegegnern stellt die »Gemeinschaft für Frieden und Aufbau« dar, die 1943 von Hans Winkler und dem aus dem KZ Theresienstadt entkommenen Werner Scharff gebildet wurde. Die von Luckenwalde, einer Kleinstadt südlich von Berlin, aus operierende »Gemeinschaft« half untergetauchten Juden und rief die Bevölkerung mit Kettenbriefen und Flugschriften dazu auf, den sinnlosen Krieg zu beenden und Widerstand zu leisten. Die Gruppe wurde im Oktober 1944 aufgespürt und die meisten Mitglieder verhaftet. Durch das Kriegsende kam es nicht mehr zu Verurteilungen. Dennoch wurden sechs Mitglieder der »Gemeinschaft « in Lagern ermordet. ${ }^{18}$

Von den schätzungsweise 10.000 bis $12.000 »$ Untergetauchten $\aleph^{19}$ in Deutschland konnte nur etwa ein Drittel überleben, davon mindestens 1.500 in Berlin. Ein Teil der Verfolgten starb bei Bombenangriffen oder an Krankheiten, andere fielen durch Anzeigen aus der Bevölkerung oder bei Ausweiskontrollen der Gestapo in die Hände. Nicht selten wurden sie in Berlin auch von jüdischen »Greifern« entdeckt, die seit März 1943 im Dienst der Gestapo versuchten, »Illegale« aufzuspüren, um dadurch ihr eigenes Leben zu schützen. ${ }^{20}$ Neben Faktoren wie Alter und körperlicher Konstitution, vermeintlich jüdischem Aussehen und materiellen Ressourcen

16 | Christine Zahn: »)Nicht mitgehen, sondern weggehen!` Chug Chaluzi - eine jüdische Jugendgruppe im Untergrund«, in: Wilfried Löhken/Werner Vathke (Hg.), Juden im Widerstand. Drei Gruppen zwischen Überlebenskampf und politischer Aktion. Berlin 1939-1945, Berlin: Edition Hentrich 1993, S. 159-205.

17 | Regina Scheer: Im Schatten der Sterne. Eine jüdische Widerstandsgruppe, Berlin: Aufbau Verlag 2004.

18 | Barbara Schieb-Samizadeh: »Die Gemeinschaft für Frieden und Aufbau«, in: Wilfried Löhken/Werner Vathke (Hg.), Juden im Widerstand. Drei Gruppen zwischen Überlebenskampf und politischer Aktion. Berlin 1939-1945, Berlin: Edition Hentrich 1993, S. 37-81.

19 | Namentlich bekannt sind mir derzeit ca. 3.400, unter ihnen 1.495 Männer und 1.845 Frauen.

20 | Siehe Doris Tausendfreund: Erzwungener Verrat. Jüdische »Greifer« im Dienst der Gestapo 1943-1945, Berlin: Metropol 2006. 
spielte auch die Geschlechtszugehörigkeit für das Leben im Untergrund eine wichtige Rolle. Die Gefahr, gefasst zu werden, war für Männer deutlich größer als für Frauen. Jeder Mann im »wehrfähigen« Alter wurde verdächtigt, Deserteur zu sein. Als im Herbst 1944 alle Männer von 16 bis 60 Jahren zum »Volkssturm« sollten, spitzte sich die Lage noch weiter zu. Jüdische Männer mussten deshalb versuchen, sich so wenig wie möglich in der Öffentlichkeit zu zeigen. Oder sie mussten über falsche Papiere verfügen, die einer eingehenden Inspektion standhielten. Wenn sie bei einer Kontrolle Verdacht erregten, so waren sie in der Regel durch ihre Beschneidung leicht zu überführen. Frauen konnten sich dagegen in der Öffentlichkeit unauffälliger bewegen und manchmal auch Arbeit finden, etwa als Haushaltshilfe, wodurch sie nicht nur etwas Geld verdienen konnten, sondern auch besser getarnt waren.

Wenn jüdische Familien jedoch mit einem oder mehreren Kindern untertauchten und die Eltern sich trennen mussten, blieben die Kinder meist bei der Mutter, was die Suche nach einem Versteck schwieriger machte als für eine einzelne Person. Insbesondere mit Kleinkindern war die Situation gefährlich, denn diese konnten unwillentlich die Tarnung verraten. Auch bestanden für Jüdinnen ganz besondere Probleme, wenn sie schwanger waren oder wurden. Schließlich konnten sie legal keine medizinische Hilfe in Anspruch nehmen.

\section{Hilfe als Form humanitären Widerstands}

Trotz aller Eigeninitiative waren die Verfolgten in der Regel auf nichtjüdische Deutsche angewiesen, die unter hohem persönlichem Risiko zur Hilfe bereit waren. Wie viele es waren, kann nur vage geschätzt werden. Bis lange nach dem Krieg, bis zum Beginn des Eichmann-Prozesses 1961 und dem zwei Jahre später in Frankfurt/Main beginnenden Auschwitz-Prozess, wurde sowohl in West- als auch in Ostdeutschland kaum über die Judenverfolgung gesprochen. Dies hatte auch zur Folge, dass weder die Helfer noch die Juden, die auf diese Weise überlebt hatten, sich ermutigt fühlten, über ihre Erfahrungen zu sprechen. Zu den Ausnahmen gehörte u. a. Marga Spiegel, die bereits 1964 ihre Erinnerungen an ihre »Retter in der Nacht« - so der Titel ihres Buches - veröffentlichte. Sie setzte sich im Übrigen auch dafür ein, dass die Bauern, die sie geschützt hatten, eine öffentliche Anerkennung erfuhren. Dies geschah allerdings nicht in Deutschland, sondern in Israel, wo sie 1969 von der israelischen Gedenkstätte Yad Vashem als »Gerechte unter den Völkern« geehrt wurden. $^{21}$

Trotz der schwierigen Quellenlage kann man die Zahl der Helferinnen und Helfer auf mehrere Zehntausend schätzen. Namentlich bekannt sind dem Ausstellungsprojekt »Stille Helden« an der Gedenkstätte Deutscher Widerstand derzeit etwa 3.200 Deutsche, die während des Zweiten Weltkrieges zur Rettung verfolgter Juden beitrugen, sei es durch Bereitstellung von Unterkünften und Nahrung, die Beschaf-

21 | Von Yad Vashem geehrt wurden Hubert Pentrop, Heinrich Silkenbömer, Bernhard Südfeld, Heinrich Aschoff und Bernhard Sickmann. Inzwischen erhielten ca. 420 Deutsche die Auszeichnung als »Gerechte« von der israelischen Gedenkstätte. 
fung falscher Papiere oder durch Fluchthilfe. Ihr Handeln widerlegt die Rechtfertigung vieler Deutscher nach dem Krieg, niemand habe gegen den Nazi-Terror etwas tun können. Angesichts von Millionen Menschen, die das Regime billigten oder zumindest nicht gegen die Judenverfolgung einschritten, ist die Zahl der Helfer gering - doch ist sie nicht so klein wie lange Zeit vermutet.

Auffallend ist, dass etwa zwei Drittel der Helfenden Frauen waren. Dies ist ein erstaunlich hoher Prozentsatz, wenn man berücksichtigt, dass der Frauenanteil am aktiven politischen Kampf unter zehn Prozent lag, was Stichproben aus den Gestapoakten von Düsseldorf und Essen ergaben, die die Historikerin Christl Wickert durchführte. ${ }^{22}$ Die Tatsache, dass ein großer Teil der männlichen Bevölkerung seit Kriegsbeginn zur Wehrmacht eingezogen war, kann den hohen Frauenanteil allein nicht ausreichend erklären. Es scheint, dass viele Frauen sich das nationalsozialistische Frauenbild, das von politischer Passivität geprägt war, zunutze machten und ihre spezifischen Handlungsspielräume zur Hilfe für Verfolgte einsetzten. So waren sie aufgrund der geschlechtsspezifischen Rollenverteilung z. B. für Lebensmittelüberbringung geeigneter als Männer, da dies bei ihnen unverdächtiger war. Man darf jedoch nicht vergessen, dass die Bewältigung dieser Aufgaben aufgrund von Rationierungen und knapper werdenden Ressourcen während des Krieges immer schwieriger wurde. Beim Besorgen zusätzlicher Lebensmittel oder falscher Papiere mussten nicht selten Methoden angewandt werden, die verboten waren oder großes logistisches Geschick erforderten. Man musste beispielsweise auf dem Schwarzmarkt handeln und hohe Preise zahlen. Trotz der Verantwortung für ihre Familien, die vor allem den Frauen oblag, gingen viele das Risiko ein und halfen Verfolgten, die sie oftmals vorher kaum gekannt hatten.

Zwar existierte zu keinem Zeitpunkt ein Gesetz im Strafgesetzbuch, das Hilfeleistungen für untergetauchte Juden ausdrücklich verbot. Die Helfer, die von der Justiz - meist von den Sondergerichten - angeklagt wurden, verurteilte man aber aufgrund anderer Delikte. So wurde etwa das Quäker-Ehepaar Carl und Eva Hermann, das Anfang 1943 ein jüdisches Ehepaar vier Wochen lang bei sich versteckt hatte, wegen »Rundfunkverbrechen« verurteilt, da es mit den Verfolgten ausländische Radiosender gehört hatte. ${ }^{23}$ In den meisten Fällen war die Ahndung entdeckter Hilfe Sache der Gestapo. Mit dem Gestapoerlass vom 24. Oktober 1941, der Kontakte von »Deutschblütigen« mit Juden kriminalisierte, wurde »Judenbegünstigung « zur strafbaren Handlung und damit sehr gefährlich. ${ }^{24}$ Das Vorgehen der Gestapo war allerdings nicht einheitlich und reichte von einer bloßen Verwarnung bis zur Haft im KZ mit Todesfolge. Es scheint, dass Frauen mit familiären Pflichten weniger hart bestraft wurden als Männer und alleinstehende Frauen. Auffallend ist jedoch, dass im Deutschen Reich die Todesstrafe nicht propagiert bzw. verhängt

22 | Christl Wickert: »Frauen zwischen Dissens und Widerstand«, in: Wolfgang Benz/ Walter Pehle (Hg.), Lexikon des deutschen Widerstandes, Frankfurt/Main: S. Fischer 1994, S. $141-156$.

23 | Dossier Carl und Eva Hermann, Gedenkstätte Deutscher Widerstand; USC Shoah Foundation Institute, Videointerview mit Eva Hermann, 12.9.1996, Nr. 19848.

24 | J. Walk: Sonderrecht, S. 353. 
wurde, um vor der Unterstützung für Juden abzuschrecken, wie es im okkupierten Polen der Fall war. Insgesamt wollten die Verfolgungsbehörden Hilfeleistungen für Juden wohl als schändliches und »abnormes« Verhalten brandmarken und hart, aber nicht allzu spektakulär bestrafen.

\section{Ganz sgewöhnlicher Deutsche}

Die Helferinnen und Helfer kamen aus allen sozialen Schichten und Milieus und halfen aus den unterschiedlichsten Gründen: sei es aus religiöser Überzeugung oder politischen Motiven, vor allem aber aus Menschlichkeit. »Ich habe nur getan, was mir mein Gewissen und meine Religion vorschrieben $«{ }^{25}$ begründete einer der Bauern seine Hilfe für Siegmund Spiegel, seinen früheren Geschäftspartner. Hier war es also, wie auch im Falle von Gertrude Sandmanns Lebensgefährtin, eine persönliche Beziehung, auf der die Hilfe basierte. In manchen Fällen beruhte sie aber auch auf einer grundsätzlichen Opposition gegen das Regime, ${ }^{26}$ während der Kontakt in anderen Fällen situativ und zufällig zustande kam. Gelegentlich gibt es auch Hinweise darauf, dass die Notlage und die Abhängigkeit der Verfolgten ausgenutzt und Geld- oder Sachleistungen gefordert wurden.

Viele Helfer empfanden ihr Handeln als selbstverständlich, als ihre menschliche Pflicht. Der lange in der Bundesrepublik vorherrschenden Auffassung folgend, verstanden sie ihr Tun meist nicht als Widerstand, d. h. als aktive, auf den Sturz des NS-Regimes gerichtete Tat. Seit den 1970er Jahren erfolgte jedoch eine begriffliche Ausdifferenzierung widerständigen Verhaltens in Formen wie Resistenz, Nonkonformität, Verweigerung und Opposition, was die Einbeziehung der Hilfe für Juden in einen solchermaßen erweiterten Widerstandsbegriff erleichterte. Mit ihrem >humanitären Widerstand ‘, mit dem sie sich außerhalb der damals kollektiv gültigen Normen stellten, haben die Helferinnen und Helfer gezeigt, dass es selbst während der NS-Diktatur und des Krieges möglich war, Menschenleben zu retten.

\section{Literatur}

Bankier, David: „Was wussten die Deutschen vom Holocaust?«, in: Kosmala/ Schoppmann (Hg.), Überleben im Untergrund (2002), S. 63-87.

Battel, Franco: »Wo es hell ist, dort ist die Schweiz«. Flüchtlinge und Fluchthilfe an der Schaffhauser Grenze zur Zeit des Nationalsozialismus, Zürich: Chronos 2000.

Fischer, Anna (Hg.): Erzwungener Freitod. Spuren und Zeugnisse in den Freitod getriebener Juden der Jahre 1938-1945 in Berlin, Berlin: Textpunkt Verlag 2007.

25 | M. Spiegel, Retter in der Nacht, S. 111.

26 | Dies war z. B. bei Elisabeth Abegg der Fall. Siehe Martina Voigt: »Grüße von >Ferdinand««. Elisabeth Abeggs vielfältiger Einsatz für Verfolgte. In: Beate Kosmala/Claudia Schoppmann (Hg.), Sie blieben unsichtbar. Zeugnisse aus den Jahren 1941 bis 1945, Berlin: Gedenkstätte Deutscher Widerstand 2006, S. 104-115. 
FU Berlin (Hg.): Gedenkbuch Berlins der jüdischen Opfer des Nationalsozialismus, Berlin: Edition Hentrich 1995.

Grossmann, Kurt R.: Die unbesungenen Helden. Menschen aus Deutschlands dunklen Tagen, Berlin: Arani Verlags GmbH 1957.

Gruner, Wolf: Widerstand in der Rosenstraße: die Fabrik-Aktion und die Verfolgung der »Mischehen« 1943, Frankfurt/Main: S. Fischer 2005.

Kingreen, Monica: "Verfolgung und Rettung in Frankfurt am Main und der RheinMain-Region«, in: Kosmala/Schoppmann (Hg.), Überleben im Untergrund (2002), S. 167-190.

Kosmala, Beate/Schoppmann, Claudia (Hg.): Überleben im Untergrund. Hilfe für Juden in Deutschland 1941-1945, Berlin: Metropol 2002.

Löhken, Wilfried/Borgmann, Karsten/Vathke, Werner (Hg.): Juden im Widerstand. Drei Gruppen zwischen Überlebenskampf und politischer Aktion. Berlin 1939-1945, Berlin: Edition Hentrich 1993.

Longerich, Peter: »Davon haben wir nichts gewusst!« Die Deutschen und die Judenverfolgung 1933-1945, München: Siedler 2006.

Mierendorff, Marta: „Von der Schweiz ausgeliefert, in Deutschland erschossen. Fam. Wohlmuth und Salomon, Deutschmeisterstr. 1«, in: Thea Koberstein/Norbert Stein (Hg.), Juden in Lichtenberg mit den früheren Ortsteilen in Friedrichshain, Hellersdorf und Marzahn, Berlin: Edition Hentrich 1995, S. 166-168.

Pretzel, Andreas/Rossbach, Gabriele (Hg.): »Wegen der zu erwartenden hohen Strafe ...«. Homosexuellenverfolgung in Berlin 1933-1945, Berlin: Rosa Winkel 2000 .

Reitlinger, Gerald: Die Endlösung. Hitlers Versuch der Ausrottung der Juden Europas 1939-1945, Berlin: Colloquium 1956.

Richarz, Monika (Hg.): Jüdisches Leben in Deutschland, Bd. 3, Stuttgart, München: DVA 1982.

Riffel, Dennis: Unbesungene Helden. Die Ehrungsinitiative des Berliner Senats 1958 bis 1966, Berlin: Metropol 2007.

Scheer, Regina: Im Schatten der Sterne. Eine jüdische Widerstandsgruppe, Berlin: Aufbau Verlag 2004.

Schoppmann, Claudia: Zeit der Maskierung. Lebensgeschichten lesbischer Frauen im »Dritten Reich«, Frankfurt/Main: S. Fischer 1998.

Dies.: »Fluchtziel Schweiz. Das Hilfsnetz um Luise Meier und Josef Höfler«, in: Wolfgang Benz (Hg.), Überleben im Dritten Reich. Juden im Untergrund und ihre Helfer, München: C. H. Beck 2003, S. 203-217.

Dies.: »Die >Fabrikaktion in Berlin: Hilfe für untergetauchte Juden als Form humanitären Widerstands«, in: Zeitschrift für Geschichtswissenschaft 2 (2005), S. $138-148$.

Dies.: »)Da packte mich das Mitleid und ich beherbergte ihn<. Tödliche Folgen für einen Bauern im Odenwald«, in: Beate Kosmala/Claudia Schoppmann (Hg.), Sie blieben unsichtbar. Zeugnisse aus den Jahren 1941 bis 1945, Berlin: Gedenkstätte Deutscher Widerstand 2006, S. 50-57.

Spiegel, Marga: »Retter in der Nacht«. Wie eine jüdische Familie im Münsterland überlebte, Köln: Pahl Rugenstein 1987. 
Tausendfreund, Doris: Erzwungener Verrat. Jüdische »Greifer« im Dienst der Gestapo 1943-1945, Berlin: Metropol 2006.

Voigt, Martina: »Grüße von `Ferdinandk. Elisabeth Abeggs vielfältiger Einsatz für Verfolgte«, in: Beate Kosmala/Claudia Schoppmann (Hg.), Sie blieben unsichtbar. Zeugnisse aus den Jahren 1941 bis 1945, Berlin: Gedenkstätte Deutscher Widerstand 2006, S. 104-115.

Wickert, Christl: »Frauen zwischen Dissens und Widerstand«, in: Wolfgang Benz/ Walter Pehle (Hg.), Lexikon des deutschen Widerstandes, Frankfurt/Main: S. Fischer 1994, S. 141-156.

Walk, Joseph (Hg.): Das Sonderrecht für Juden im NS-Staat, Heidelberg: C. F. Müller 1981.

Zahn, Christine: «)Nicht mitgehen, sondern weggehen!‘ Chug Chaluzi - eine jüdische Jugendgruppe im Untergrund«, in: Wilfried Löhken/Werner Vathke (Hg.), Juden im Widerstand. Drei Gruppen zwischen Überlebenskampf und politischer Aktion. Berlin 1939-1945, Berlin: Edition Hentrich 1993, S. 159-205. 